\title{
Primary central nervous system lymphoma: A case report
}

\author{
Cristina Caciuc ${ }^{1}$, Razvan Motoc ${ }^{1}$, Mihaela Popescu ${ }^{3}$, Alexandra Oprisan ${ }^{1,2}$ \\ ${ }^{1}$ Department of Neurology, Colentina Clinical Hospital, Bucharest, Romania \\ 2Department of Neurology, "Carol Davila" University of Medicine and Pharmacy, Bucharest, Romania \\ ${ }^{3}$ Department of Hematology, Colentina Clinical Hospital, Bucharest, Romania
}

\begin{abstract}
Primary central nervous system lymphoma (PCNSL) represents an extranodal non-Hodgkin lymphoma, limited to the central nervous system. It has an aggressive course, and despite regimens of high-dose chemotherapy, the survival rate is low. Authors report the case of a 58 year old woman with a subacute dementia and a suggestive brain MRI and CSF for central nervous system lymphoma, with histopathological confirmation of a diffuse large B-cell lymphoma - non-Hodgkin lymphoma. After chemotherapy and radiotherapy, she developed an infectious eruption: right herpes zoster involving mandibular division of trigeminal nerve. Following the eruption, the recurrence of her neurologic symptoms was observed - the possibility of a viral meningoencephalitis was considered. PCNSL requires a long term management, better treatment strategies (that prolong survival) being available in the last decade.
\end{abstract}

Keywords: primary central nervous system lymphoma, diffuse large B-cell lymphoma - nonHodgkin lymphoma, subacute dementia, high-dose methotrexate

\section{INTRODUCTION}

Primary central nervous system lymphoma (PCNSL) represents an extranodal non-Hodgkin lymphoma, involving the neuraxis (brain, eyes, leptomeninges, spinal cord), without evidence of systemic involvement (1). The most common morphology consists primarily of diffuse large CD 20+ B-cell aggregates confined to the CNS (2). It has specific characteristics, aggressive course and unsatisfactory outcome (3). Currently, there are two main therapeutic options: chemotherapy regimens based on high-dose methotrexate and whole-brain radiotherapy, however, the toxicity associated with these treatments can lead to serious age-related neurotoxicity (4). Optimal treatment approaches for patients with PCNSL remain challenging, the prognosis for patients that have failed first-line therapy remaining poor (2).

\section{CASE STUDY}

We present the case of a 58 years old woman, with a past medical history of arterial hypertension and dyslipidemia, and a family history of two relatives (first-degree and second-degree relatives) with Alzheimer's Disease, who was admitted in our department in January 2017, after being brought by her family for behavioral disturbances, memory and attention impairment, with insidious onset in the last 4 months.

In December 2016 she was examined by a clinical neurologist and an enhanced brain MRI was performed which showed cerebral atrophy and an hyperintense FLAIR area, without contrast enhancement in the splenium of corpus callosum.

On admission in our department two weeks later, general examination was unremarkable. The neurologic exam revealed a conscious, vigilant pa- 
tient, without signs of meningeal irritation, without signs of cranial nerves pathology, no motor deficit, with symmetrical deep tendon reflexes, with no pathological reflexes. The patient had no superficial or deep sensitivity disturbances. She had a MMSE (mini mental state examination) of $23 / 30$, MoCA (Montreal Cognitive Assessment) 21/30, ACE-III (Addenbrooke's Cognitive Examination) attention $15 / 18$, memory $12 / 26$, fluency $10 / 14$, language $23 / 26$, visuo-spatial15/16. She was partial temporal oriented, spatial oriented, with cognitive decline and behavioral disturbances, which consisted in stereotyped and inappropriateness of behavior, lack of inhibition (Moria), impulsivity, neglect of personal hygiene, loss of social awareness, difficulty in organizing and executing tasks (dinners). She also had hypoprosexia, inappropriate emotional reactions, absence of empathy and memory impairment.

Laboratory blood evaluation revealed normal cell and platelet counts, without inflammatory syndrome. Biochemical laboratory serum values, coagulation parameters, TSH (thyroid-stimulating hormone), free-T4, thyroid peroxidase antibodies, vitamin B12, serum calcium were within the normal range. A serological screen for syphilis and HIV (human immunodeficiency virus) was conducted, with negative results.

The electroencephalography (EEG) did not reveal any pathological discharges.

We performed a lumbar puncture-which revealed normal appearance and normal opening pressure of the cerebrospinal fluid (CSF), negative
Pandy's test, with normal glucose concentrations, a raised protein level $(57,8 \mathrm{mg} / \mathrm{dl})$, with mild pleocytosis with lymphocytic predominance (7 white blood cells - lymphocites, without abnormalities in cellular immunophenotyping by flow citometry). Her CSF was negative for 14-3-3 protein. The possibility of fronto-temporal dementia and early Alzheimer disease was considered and she was tested in CSF for phosphorylated tau protein and $42 / 40$ amyloid $\beta$, with negative results for early Alzheimer disease (p-tau 28 pg/ml - NV $<61$ pg/ml; 42/40 A $\beta$ 1,1- NV > 0,5).

Considering the clinical setting (the age, the clinical examination) and the final results of the tests performed, fronto-temporal dementia could not be excluded, and a follow-up with brain MRI was recommended.

Two months later, another contrast enhanced brain MRI was performed, which revealed fronto-temporo-parietal and hippocampal atrophy, cerebellar atrophy, bilateral infiltrative lesions, centered in the genus and splenium of corpus callosum, with anterior extension into both frontal lobes and posterior extension into right occipital lobe, hypointense on $\mathrm{T} 1$ weighted imaging with petechial contrast enhancement (irregular, with nodular components), hyperintense on $\mathrm{T} 2$ weighted imaging and FLAIR, with restricted diffusion on DWI (diffusion-weighted imaging) and normal ADC (apparent diffusion coefficient) values, with little mass effect and limited surrounding vasogenic edema (Fig. 1, 2).
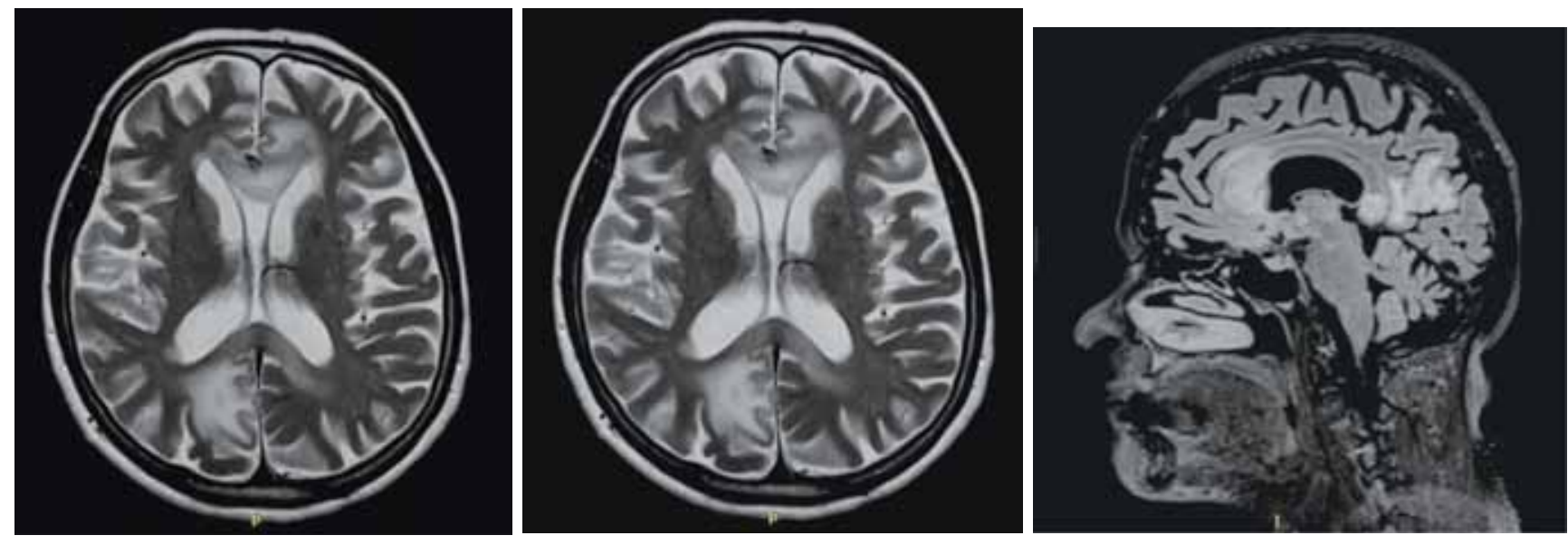

FIGURE 1. Axial T2W-MRI (A), axial FLAIR (B), sagittal FLAIR (C) images showing 2 localized, large, bilateral, asymmetrical lesions centered in the genus and splenium of the corpus callosum, with extension mainly 


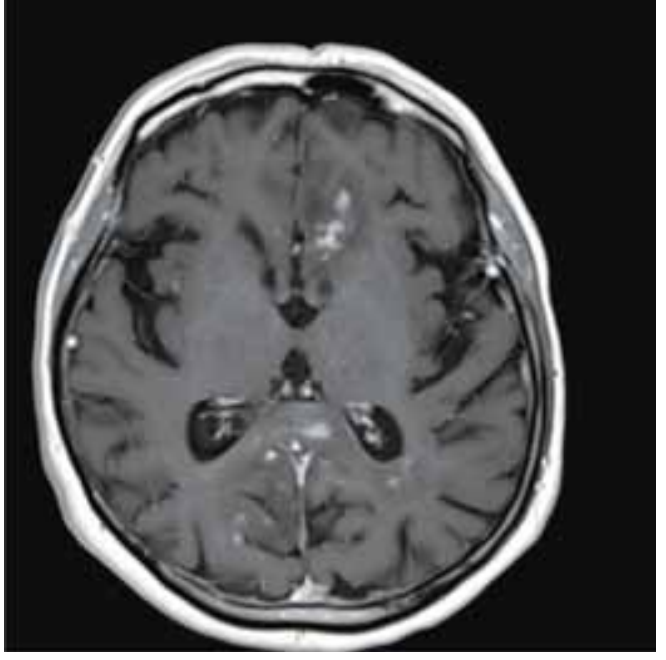

FIGURE 2. Axial T1+C $(G d)$-weighted MRI demonstrates diffuse contrast enhancement, with nodular components

Brain MRI was highly suggestive of a lymphomatous proliferative process. A stereotactic needle biopsy was necessary to confirm the diagnosis - it demonstrated perivascular, atypical lymphocytic infiltration, with histopathological and immunostaining examinations characteristic of diffuse large B-cell lymphoma - non-Hodgkin lymphoma with $\mathrm{CD} 20+, \mathrm{CD} 79 \mathrm{a}+$ diffuse, large B-cell.

She was referred to a hematologist, and a CTscan of the chest, abdomen and pelvis was performed for exclusion of the systemic involvement. It did not show the presence of lymphoma elsewhere and a diagnosis of primary central nervous system lymphoma with diffuse, large B-cell was established. Also an ophthalmologic evaluation with fundoscopy completed the diagnostic procedure - it did not reveal lymphomatous involvement of the retina. At one month follow-up she developed left hemiparesis (4/5) and still had behavioral changes and cognitive impairment.

She began treatment with methotrexate (MTX) and cytarabine (Ara-C) (Ferreri/IELSG protocol), preceded by intrathecal MTX and dexamethasone administration. At 3 months follow-up: a slight amelioration of her left hemiparesis $(+4 / 5)$ and cognitive performance were noticed, but she still displayed behavioral disturbances.

The consolidation therapy after high-MTX based chemotherapy was performed with wholebrain radiotherapy, with a total dose of $36 \mathrm{~Gy}$, standard fractonation. Further, her behavioral disturbances submitted. A week after completion of the radiotherapy, the family observed a cutaneous eruption - right herpes zoster involving mandibular division of trigeminal nerve. Following the eruption, the family remarked, in a couple of days, the recurrence of behavioral disturbances, attention deficit, memory impairment. A contrast enhanced brain MRI was performed -it did not show signs of disease relapse or new focal lesions.

She was again admitted in our department. Neurologic exam revealed a partial temporal oriented, spatial oriented patient, with cognitive decline and behavioral disturbances - stereotype and inappropriate behavior (symptoms that existed in the past, but submitted after specific therapy), with attention deficit and memory impairment (MMSE 24/30, MoCa 18/30, ACE III: attention 16/18, memory $17 / 26$, fluency $8 / 14$, language $24 / 26$, visuo-spatial14/16), a slight gait ataxia and left leg monoparesis (residual).

Laboratory blood evaluation was unrevealing. CSF evaluation showed a slight raised protein level (48 mg/dl), with normal cell count (4 white blood cells - lymphocytes) and normal glucose level, without abnormalities in cellular immunophenotyping by flow citometry, bacterial and fungal culture negatives.

In view of her cognitive impairment and recent infectious eruption, the possibility of a viral meningoencephalitis was considered. A lymphomatous reoccurrence or an adverse reaction of intrathecal chemotherapy/radiotherapy could not be excluded. The patient was examined by an infectious diseases physician and a hematologist and received high dose antibiotherapy and antiviral therapy (acyclovir, ceftriaxone, ampicilin) for 7 days, when a lumbar puncture was performed, which showed a decreased in pleocytosis. She also received low dose dexamethasone with vitamin B.

She was discharged 14 days later. Her behavioral disturbances gradually, but markedly improved, and a slight amelioration was noticed in her memory function (MMSE 26/30, MoCA 24/30). At 3 months follow-up, her behavioral disturbances submitted and she had mild memory impairment and left hemiparesis. One and a half year after the onset of her neurological illness, our patient was evaluated and was found to have improved cognitive function and left hemiparesis. 


\section{DISSCUSION}

Primary central nervous system lymphoma (PCNSL) represents an extranodal, non-Hodgkin lymphoma, limited to central nervous system (brain, leptomeninges, eyes, spinal cord) in the absence of systemic diffusion (1) (2). It accounts for $1 \%$ of all lymphomas and $2-5 \%$ of primary CNS tumors (1). More than $90 \%$ of PCNSL are diffuse large B cell lymphoma, with more than half cases with intracerebral localisation $(1,2)$ - multifocal involvement is seen in more than half cases, but leptomeningeal, ocular involvement and rarely spinal involvement can be detected (1).

The overall incidence is $0.44 / 100000$ persons $(5,6)$, with a high incidence in immunocompromised patients - patients with both congenital and acquired immunosupression (HIV and post-transplant conditions) have increased risk of PCNSL (1). Presentation in these patients is in their $3^{\text {th }}-4^{\text {th }}$ decades. Immunosuppression (acquired/congenital) is considered to be a risk factor for the development of PCNSL - therefore a serologic screening for HIV infection is mandatory in these patients $(1,4)$.Recently, there has been an increase of PCNSL incidence in immunocompetent individuals, especially in older patients $\left(5^{\text {th }}-7^{\text {th }}\right.$ decades $)(1)$, with a median age at diagnosis in the fifth decade. Our patient was a 58 year-old immunocompetent female.

The pathogenesis of PCNSL is still unclear. Epstein-Barr virus (EBV) may have a causal link to the development of PCNSL in immunocompromised patients, possibly as a consequence of proliferation of B-lymphocytes by viral stimulation and due to a lack of suppressive T-cells activity in a immunosuppressive setting (2). More than $95 \%$ of PCNSL express B-cells markers: CD20+, CD19+, CD 79a+ (2).

Patients with PCNSL develop progressive neurologic signs over weeks, often with subacute onset, including focal neurologic deficits (56\%-70\%), mental status and behavioral changes (32-43\%), symptoms of increased intracranial pressure (33\%) and/or seizures (11-14\%), depending on the site of CNS involvement (3). Less frequently eyes, and rarely, spinal cord are involved $(1,3)$. Leptomeningeal involvement can also be detected $(1,4)$. Ocular symptoms (blurred vision, decreased visual acui- ty), due to involvement of retina, choroid or vitreous, can be either isolated or coexist with cerebral symptoms -about $50 \%$ of cases with PCNSL and ocular involvement have no visual symptoms - an ophthalmologic evaluation with fundoscopy and slit lamp examination is necessary in all patients with PCNSL (1-3).

Brain MRI is the imaging study used in order to define site and extension of the disease (4). Typical findings, in immunocompetent patients, are homogenously enhancing single $(70 \%)$ or multiple lesions $(30 \%)$, with periventricular space involvement $(60 \%)$, mainly supratentorial located, with modest surrounding edema and little mass effect $(2,4)$, isointense or hypointense on T1-weighted images and hyperintense on T2-weighted images and FLAIR (3). It frequently crosses the corpus callosum, usually located in periventricular areas and/ or deep grey matter (1). In our immunocompetent patient, brain MRI showed heterogeneously enhancing lesions (irregular, with nodular components), as seen in immunocompromised patients and in low grade PCNSL. Low-grade tumors (defined by the predominance of small, mature lymphocytes and a growth fraction $<20 \%$ ) differ from the high-grade PCNSL by following: deep locations are more common and contrast enhancement can be absent, irregular or mild (7).

A lumbar puncture must be done if not contraindicated - CSF should be assessed by cytology and flow cytometry (1). The CSF evaluation of our patient showed a low cell number $\left(7 / \mathrm{mm}^{3}\right)$, without detection of malignant cells. CSF, in these patients, is characterized by raised proteins levels and mild pleiocytosis, lymphoma cells being detected in 10$30 \%$ of the cases, and secondary CSF involvement is seen in $5-20 \%$ of cases (1) (2). Cellular immunophenotyping by flow cytometry in the CSF helps distinguish malignant cells from reactive lymphocytes, but low cell numbers in the CSF sample are frequently found (2).

Following MRI and CSF assessment, histopathological confirmation should be performed by resection or stereotactic needle biopsy (2-4). Immunochemistry is also required - B-cell PCNSL express B-cell lineage markers (CD20+, CD19+, $\mathrm{CD} 79 \mathrm{a}+$ ) (4). Prior to biopsy, attention should be paid to the use of glucocorticoids -it is usually recommended to defer corticoids one week before bi- 
opsy (PCNSL is highly sensitive of corticoids, through a specific cytotoxic activity on lymphoma cells) $(1,2,4)$.

To exclude the presence of the disease elsewhere and to specify the extent of the lymphoma a CTscan of the chest, abdomen and pelvis, a bone marrow biopsy (as well as a testicular ultrasound in elderly males) are necessary $(2,4)$. In our patient, we excluded a lymphomatous disease elsewhere by performing a CT-scan of chest, abdomen and pelvis.

Treatment of PCNSL includes two phases: induction and consolidation $(2,6)$. Induction phase consists of high-dose methotrexate (HD-MTX) based polychemotherapy (a significant high rate was observed with HD-MTX+cytarabine) (2-4) our patient received HD-MTX with cytarabine, with a slight amelioration of her neurological status. Rituximab was added, with neurologic improvement - slight amelioration of behavioral disturbances and improvement of memory impairment (1). The addition of rituximab to HD-MTX chemotherapy improves overall survival rate, complete response, reduces nervous system relapses, without supplementary neurotoxic effects $(2,3)$. Complete response rate after initial chemotherapy is $40-60 \%$, however, about half of these cases will relapse, needing consolidation $(1,4)$.

For consolidation phase, there are two choices - whole-brain radiotherapy (WBRT) or high dose chemotherapy with autologous stem cells transplantation (HD-ASCT) $(1,6)$. Our patient was not eligible for HD-ASCT, because she still displayed behavioral disturbances. WBRT was preferred. It is associated with short lasting responses and relapses, delayed treatment-related neurotoxicity (impaired psychomotor speed, executive function, memory and attention) - imaging demonstrating leukoencephalopathy and subcortical/cortical atrophy $(1,3)$. In our patient, these side effects did not occur at one month follow-up.

Relapses may be due to seeding from distant CNS sites, ocular disease, distal to the initial site of presentation (9). Patients without signs of active clinical/radiological disease are at risk of relapses after 5 years of remission (9).

In patients with recurrence of neurologic symptoms after intrathecal chemotherapy and radiotherapy, the differential diagnosis with infectious dis- eases, disease relapse, or a side effect of intrathecal methotrexate/radiotherapy must be done. In our patient we considered acute radiation effects slightly improbable-brain swelling may be seen on brain MRI, but without restricted diffusion on DWI. In PCSNL relapse, one may observe new lesions on brain MRI, with or without contrast enhancement, with restricted diffusion on DWI, and abnormalities in cellular immunophenotyping by flow citometry on CSF evaluation (10). Our patient had no new lesions on brain MRI, and no abnormalities in cellular immunophenotyping by flow citometry. No infectious agent could be demonstrated, but a therapeutic response was obtained and made this hypothesis more likely.

In PCNSL patients, survival is poor - prognosis has significantly improved as a result of better treatment strategies, but these are not curatives (they increased overall survival with 44 months) $(1,2)$. Overall survival rates were $53 \%$ at 1 year, $38 \%$ at 2 years, and $26 \%$ at 5 years after diagnosis (2). There are 3 prognostic systems: IELSG (International Extranodal Study Group) score being the most commonly used - independent poor predictors are: age $>60$ yrs, ECOG (Eastern Cooperative Oncology Group) performance status $>1$, elevated serum level LDH, elevated protein level of CSF and involvement of deep regions of the brain (1). Two year survival rates have been found as $80 \%$ in patients with 0-1 factor (as seen in our patient), $48 \%$ in patients with $2-3$ factors, and $15 \%$ in patients with 4-5 factors (1). Younger age and higher Karnofsky performance score at the time of diagnosis are believed to be associated with prolonged survival time (9), this being the case of our patient.

\section{CONCLUSIONS}

PCNSL is a rare subtype of extranodal non-Hodgkin lymphoma and should be considered in a young patient with subacute progressive dementia. In these patients, brain enhanced MRI and CSF examination are mandatory, along with ophthalmologic evaluation with fundoscopy (searching for intraocular lymphoma), serologic screening for HIV infection (considered to be a risk factor), and CT scan of chest, abdomen and pelvis (for staging). The gold standard remains stereotactic needle biopsy, with deferring corticosteroids one week prior to biopsy. 
Over the last decades significant progress in the treatment of patients with PCNSL has been made, with improved survival. HD-MTX based chemotherapy is currently considered the standard induc- tion treatment. The current focus is to reduce the number of refractory cases and to prolong remission.
Conflict of interest: none declared
Financial support: none declared

7. Jahnke K., Schilling A., Heidenreich J. et-al. Radiologic morphology of low-grade primary central nervous system lymphoma in immunocompetent patients. AJNR Am J Neuroradiol. 2005; 26:2446-54.

8. Ambady P., Fu R., Netto J.P., Kersch C. et al. Patterns of relapse in primary central nervous system lymphoma: inferences regarding the role of the neuro-vascular unit and monoclonal antibodies in treating occult CNS disease. Fluids Barriers. 2017; 14.

9. Walker A., Ruzevick J., Malayeri A. et al. Postradiation imaging changes in the CNS: how can we differentiate between treatment effect and disease progression? Future Oncol. 2014; 10: 1277-1297.

10. Szczepanek D., Wąsik-Szczepanek E., Stoma F. et al. Primary central nervous system lymphoma as a neurosurgical problem. Neurol Neurochir Pol. 2017.s
6. Qian L., Tomuleasa C., Florian I.A. et al. Advances in the treatment of newly diagnosed primary central nervous system lymphomas. Blood research. 2017; 52 